\title{
GCU
}

Glasgow Caledonian

University

University for the Common Good

\section{An imaging system for visual inspection and structural condition monitoring of railway tunnels}

Jenkins, Mark David; Buggy, Tom; Morison, Gordon

Published in:

2017 IEEE Workshop on Environmental, Energy, and Structural Monitoring Systems (EESMS)

DOI:

10.1109/EESMS.2017.8052679

Publication date:

2017

Document Version

Author accepted manuscript

Link to publication in ResearchOnline

Citation for published version (Harvard):

Jenkins, MD, Buggy, T \& Morison, G 2017, An imaging system for visual inspection and structural condition monitoring of railway tunnels. in 2017 IEEE Workshop on Environmental, Energy, and Structural Monitoring Systems (EESMS). IEEE, IEEE Workshop on Environmental, Energy, and Structural Monitoring Systems (EESMS), Milan, Italy, 24/07/17. https://doi.org/10.1109/EESMS.2017.8052679

\section{General rights}

Copyright and moral rights for the publications made accessible in the public portal are retained by the authors and/or other copyright owners and it is a condition of accessing publications that users recognise and abide by the legal requirements associated with these rights.

Take down policy

If you believe that this document breaches copyright please view our takedown policy at https://edshare.gcu.ac.uk/id/eprint/5179 for details of how to contact us. 


\title{
An Imaging System for Visual Inspection and Structural Condition Monitoring of Railway Tunnels
}

\author{
Mark David Jenkins, Tom Buggy \& Gordon Morison \\ School of Engineering and Built Environment \\ Glasgow Caledonian University \\ Scotland, United Kingdom \\ Email: gordon.morison@gcu.ac.uk
}

\begin{abstract}
This paper details a complete hardware and software system designed to aid in the visual inspection and structural condition monitoring of railway tunnels. The system consists of two main components; an image acquisition system for data collection and an image processing software package for data analysis. The image acquisition system consists of an array of cameras with overlapping fields of view, a uniform lighting solution and a computer to control recording mounted on a rail trolley. The software package carries out operations such as image registration and stitching, 3D reconstruction and change detection. This software package is designed to generate and present image information in a manner which is most useful to structural examiners. The overall system aims to reduce the time taken to carry out visual inspection of tunnels while increasing the overall accuracy of inspections. Our preliminary findings indicate that the system directly benefits examiners when carrying out visual tunnel inspections.
\end{abstract}

\section{INTRODUCTION}

The railway tunnel infrastructure is a vast network upon which society has become heavily reliant. The proper inspection and monitoring of these tunnels is incredibly important in ensuring that failures are avoided. This is important from a safety aspect as well as a financial point of view as unscheduled closures of tunnels due to failure can be incredibly costly to transport operators. Many of the tunnels used for transport purposes today are now operating well past their intended lifetime at the time of construction and are also subject to an ever increasing volume of traffic. This increases the requirement for careful monitoring to ensure that they remain fit for purpose. The majority of the inspection work carried out on the tunnel infrastructure is done manually and is therefore time consuming, due to the inaccessibility of certain areas an the frequent need for scaffolding, and subject to human error in an environment where visibility is often poor. This has lead to the need for a more robust, less time consuming method for tunnel inspection.

In order to carry out predictive maintenance on the tunnel infrastructure to avoid unforeseen closures, there are a number of visual indicators which are monitored by inspection teams. These indicators include surface cracks, surface corrosion, open joints in brickwork and water leakage. The detection and monitoring of these indicators, which is carried out manually in the majority of cases, is paramount in the evaluation of tunnel safety and allows for early maintenance of possible faults which is financially beneficial.
This paper presents a combined hardware and software system to aid examiners in the visual inspection and structural condition monitoring of tunnel infrastructure. The hardware system consists of an overlapping array of cameras and a uniform lighting solution which is used to collect visual data from the interior of a tunnel. The software package then conducts analysis of this data, the result of which is presented to examiners to aid in their inspection and reporting. The system presents a number of benefits to examiners, the most useful of which is the direct comparison of current data to the data gathered in previous examinations. This is carried out using a change detection algorithm which directs examiners to areas of interest, allowing them to view the exact extent of the detected change over the time period between inspections. A Structure from Motion (SfM) algorithm is also utilised to generate 3D models of the tunnels to provide spatial context which is useful in classifying the severity of a given fault.

The remainder of this paper will discuss related work (Section II), the types of faults targeted during an inspection (Section III), the proposed hardware solution for acquisition of the tunnel data (Section IV) and the details of the various software components which are utilised to aid in examination (Section V). An evaluation of our overall system and how it has impacted inspection and our concluding remarks are then given in Section VI.

\section{RELATED WORK}

There are a small number of systems available at present for tunnel lining inspection. Systems such as [1] and [2] utilise a combination of imaging systems and laser scanning. The use of laser scanning gives highly precise measurement of the tunnel interior which is not strictly a requirement for successful inspection and makes the systems very costly. Laser scanning alone [3] produces an accurate point cloud but lacks the image data which is much more useful in this particular application than the accurate dimensional measurements provided. The system proposed in this paper is more cost effective while providing all of the information necessary to perform a successful tunnel inspection. The work by Stent et.al [4] also aim to provide a more economic solution but in a more constrained environment. This system focuses on single bore tunnels with centred tracks such as subway tunnels. This simplifies the problem as the camera array can be positioned in 


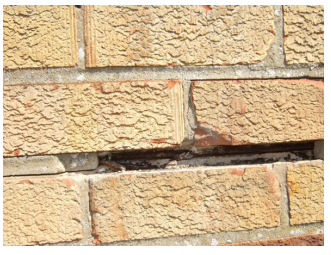

(a) Open Joint in Brickwork

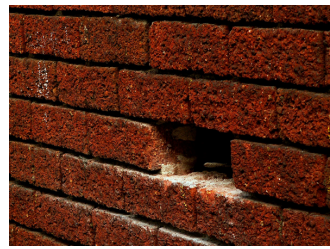

(b) Missing Brick

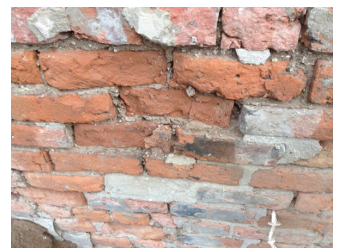

(c) Spalling or Surface Corrosion

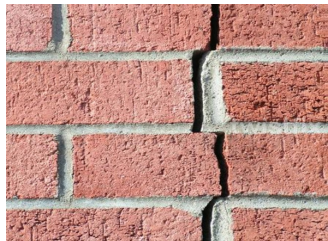

(d) Cracked Brickwork

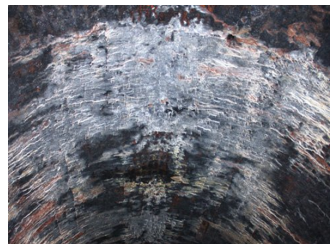

(e) Calcite Deposit from Water Ingress

Fig. 1. Examples of various defects which examiners look for when conducting an inspection

the centre of the tunnel which is not possible in most railway tunnels with multiple tracks. As the system is operating in the centre of single track tunnels it also assumes that the geometry of the tunnel is cylindrical during processing. The work in this paper does not rely on such assumptions and is designed to be utilised in a range of tunnels of varying geometry with multiple tracks.

\section{TARGETED FAULTS}

There are a number of faults that structural examiners look for when inspecting a tunnel. In order to create a system fit for use in the inspection process, all of these faults must be taken into consideration. The main faults which must be considered are listed below and an example of each is given in Figure 1.

- Open Joints: This indicates a deterioration of the mortar between the brickwork on the lining of the tunnel (Figure 1(a))

- Missing Bricks: A collection of bricks, a single brick or part of a brick is missing from the tunnel lining (Figure 1(b))

- Spalling: Surface corrosion of the brickwork on the tunnel lining (Figure 1(c))

- Cracks: A visible split in the material lining the tunnel, potentially running directly through bricks or confined to the mortar (Figure 1(d))

- Water Ingress: Visible water on the interior of the tunnel lining or evidence of previous water intrusion such as calcite deposit (Figure 1(e)) or moss growth

Due to the nature of the faults listed previously, any worsening will result in a change which is visible on the lining of the tunnel. This validates the principal behind a visual change detection system and shows that it is ideal for assisting examiners in their inspection. Detected changes can be brought to the attention of the examiner who can then assess the severity of the change.

\section{HARDWARE SOLUTION}

This section will discuss the considerations made when developing the hardware system which is used for the collection of image data within the tunnel infrastructure. As the system was designed to be utilised directly with a specific software package, the functionality of the software package lead to a number of conditions that the acquisition system must fulfil. This produced the following conditions for the acquisition system:
1) Images must be acquired around the full circumference of the tunnel lining with sufficient overlap for image registration and Structure from Motion (SfM)

2) Images must be acquired along the entire length of the tunnel with sufficient overlap for image registration and Structure from Motion (SfM)

3) Lighting must be uniform across the image to avoid introducing shadow errors

The final image acquisition system which was designed fulfils all of these conditions. A total of 5 GigEVision cameras are positioned in an array where the field of view of each camera overlaps with the next camera. The cameras are positioned such that they cover approximately half of the interior of the tunnel as shown in Figure 2. This obviously does not immediately fulfil condition 1 above but it was decided that a two-pass image acquisition approach had multiple benefits. The first of these relates to the position of the system within the tunnel. The majority of tunnels contain more than one track and as a result, the hardware system cannot be placed directly in the centre of the tunnel. This means that the cameras would be at a much greater distance from one wall than the other resulting in a potentially large difference in resolution between the images. Secondly, there is a possibility of obstructions in the centre of the tunnel between the tracks. This means that conducting image acquisition from a single track may result in some images of the tunnel lining being obscured. By positioning the cameras as shown in Figure 2 and conducting

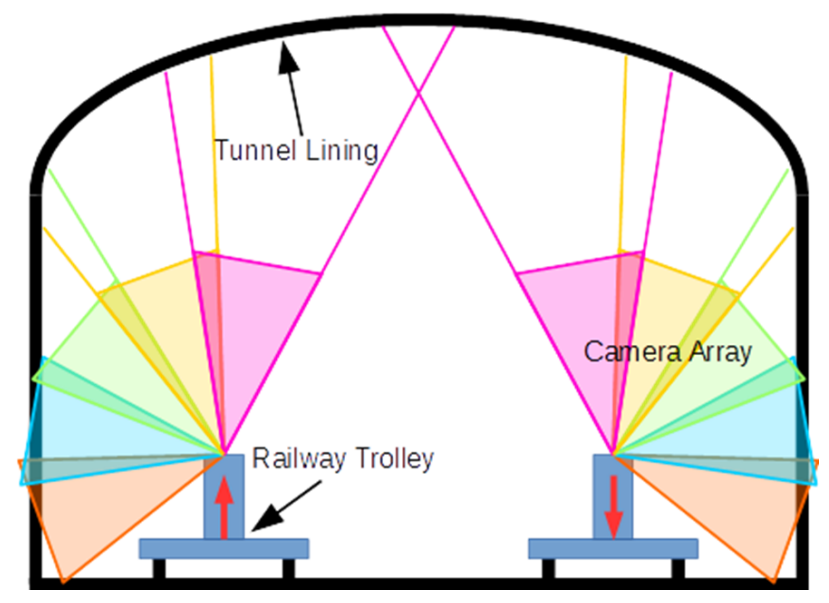

Fig. 2. Diagram of camera array with overlapping cameras scanning a tunnel in two passes where the red arrow shows the direction of motion 


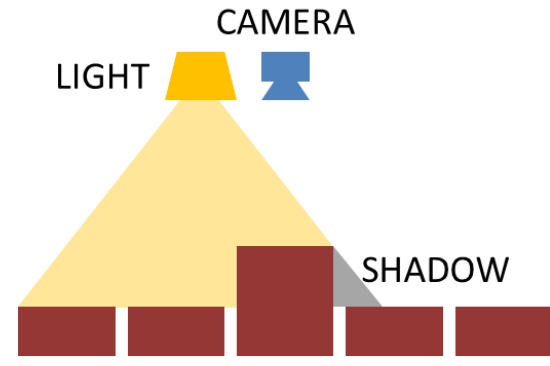

(a) Protrusion on surface of tunnel causes shadow which is detected by camera

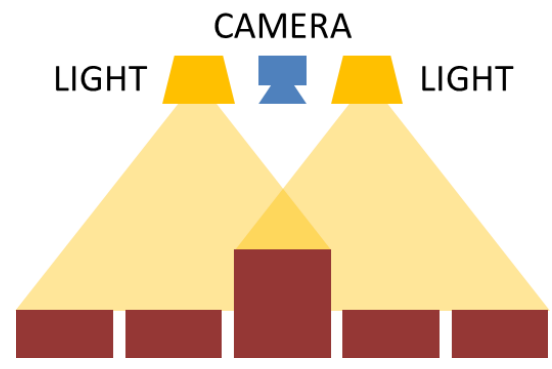

(b) Secondary light removes the shadow created by the single light

Fig. 3. Illustration of the shadow issue caused by a single light and how this can be avoided using two points of illumination

image acquisition in two stages it is ensured that the highest quality data of the entire tunnel interior is obtained. This completely satisfies condition 1 above.

Condition 2 relates to the frame-rate at which image acquisition is carried out and the speed at which the system is moved through the tunnel. The system is moved at a walking pace through a tunnel. Condition 2 is satisfied by ensuring that the frame-rate of the cameras is high enough to result in image overlap at this speed.

During experimentation with various lighting solutions it was discovered that the position of the lighting had a significant impact of the detection of changes in the images. As shown in Figure 3, positioning a single light source at an offset position from the camera resulted in the potential for minor shadows to be cast which were often detected as changes in the tunnel lining increasing the false positive rate. To reduce the impact that the lighting solution had on the change detection algorithms, each camera was fitted with a pair of lights; one in front and one behind. Each light counteracts the shadows from the other giving a more uniform light to the area where image acquisition is taking place thus satisfying condition 3 of the specification.

A basic block diagram of the final image acquisition system is given in Figure 4 which shows the rail trolley, power supply, recoding computer, PoE switch, lighting and camera array. The power supply is a generator capable of powering the computer, lights and PoE switch. All cameras are PoE compliant and therefore require only one connection for both data and power and the trolley is a standard railway trolley.

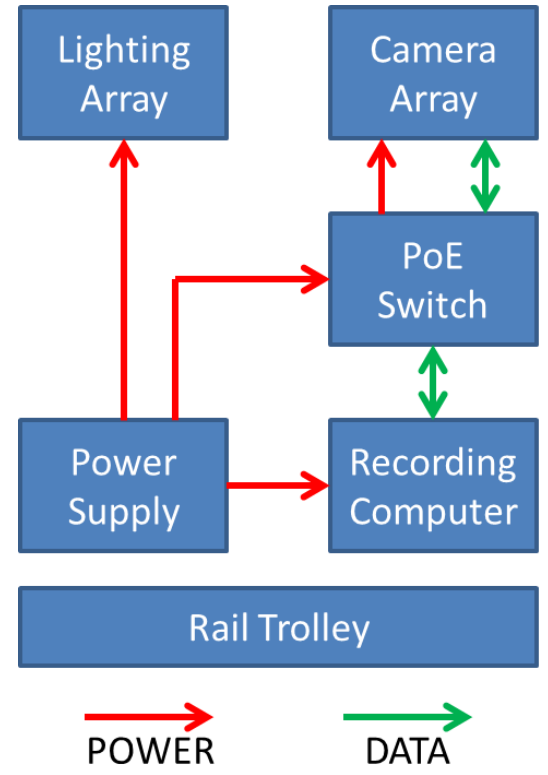

Fig. 4. Block diagram of how the hardware system is connected for on site image acquisition

\section{SOFTWARE SOLUTION}

As discussed previously, the purpose of the software package is to analyse the images collected by the hardware system and generate information which is useful to structural examiners carrying out inspection of the tunnel. There are three main components to the software package; image stitching, change detection and 3D reconstruction. Each of these sections are discussed below with focus given to the benefit of the result to the structural examiner.

\section{A. Image Stitching}

A key factor of classifying a defect is the spatial context of that defect. The severity of a crack for example depends on its spatial position within the tunnel as well as its physical attributes. While a single image of a defect can reveal its physical characteristics, more information on the spatial position of the defect is required for the examiner to make an informed assessment. For this reason a 2D image stitching algorithm is implemented [5], [6], [8]. This algorithm takes the individual images captured by the hardware system and, through a series of feature extraction, warping and alignment techniques, creates a single $2 \mathrm{D}$ image showing the entire tunnel surface. This is shown in Figure 5 where a small set of images covering a section of tunnel (Figure 5(a)) is used to generate the larger stitched image (Figure 5(b)). Examiners can then use this larger image to view defects in context which allows for more accurate assessment of the tunnel condition.

\section{B. Change Detection}

Many tunnels run for extensive distances and there can be large sections of tunnel lining which remain completely unchanged between inspections. The manual method of tunnel 


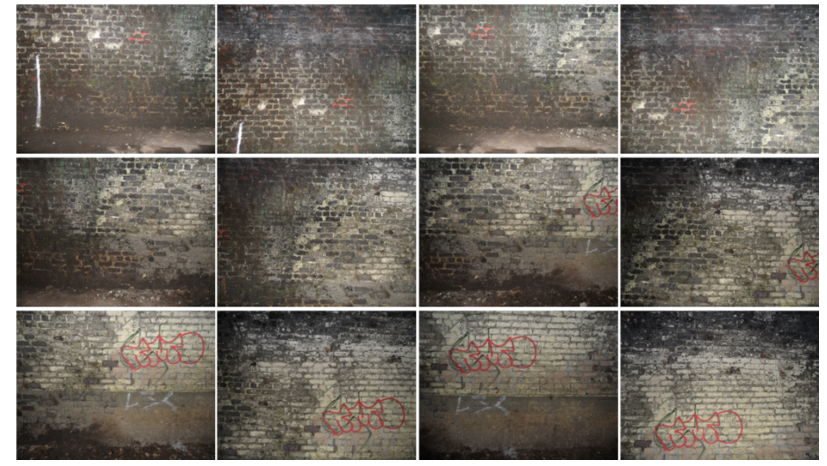

(a) Individual images covering an area of tunnel

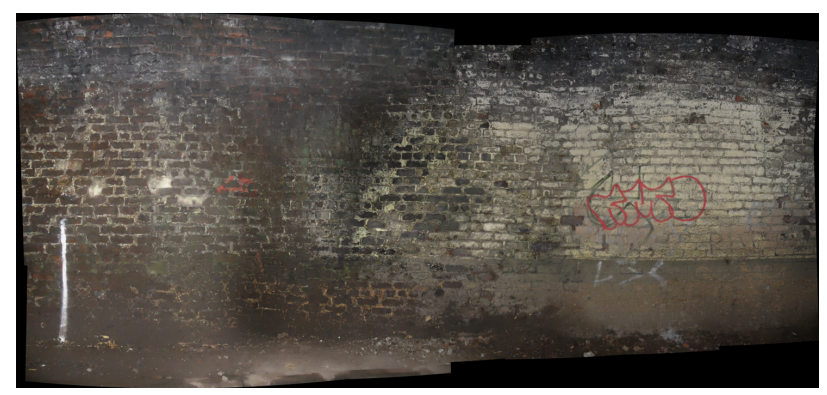

(b) Stitched image showing the complete area in one image

Fig. 5. A selection of images covering an area of tunnel lining and the resultant stitched image

inspection means that a large amount of time is wasted conducting inspection of unchanged structure. Directing examiners to the locations at which a change has occurred is vastly more efficient and means that time does not have to be wasted inspecting images of areas which have not changed since the previous inspection. The change detection [7] system employed in the software package achieves this increase in efficiency by detecting the location of changes and directing the examiner to the images containing the defects which must be monitored.

As shown in Figure 6, the change detection is carried out in 3 main stages. Given an image from a previous scan as the template image (Figure 6(a)), the best matched image from the current scan is selected and used as the query image (Figure 6(b)). Due to the nature of the image acquisition system it is highly unlikely that two images will be captured which overlap exactly. This means that the query image must first be aligned with the template image before changes can be detected. Using the same feature extraction, image warping and alignment techniques utilised by the image stitching algorithm, the query image is aligned with the template image (Figure 6(c)). Now that the template and query images are accurately aligned, normalised cross correlation [8] based filtering can be applied to detect pixel level differences between the images. This method is more robust to minor misalignments than comparing single pixels as it takes into account surrounding pixels and also corrects for variation in lighting intensity through mean based intensity normalisation. Despite this robustness there is often still some stray pixels which are incorrectly detected

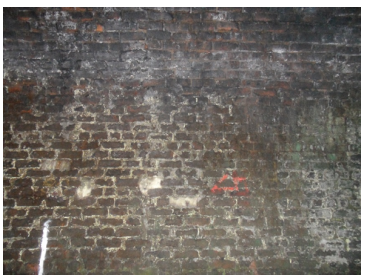

(a) Template Image

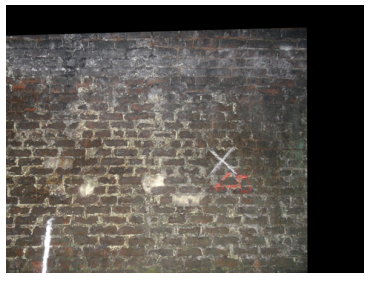

(c) Registered Query Image

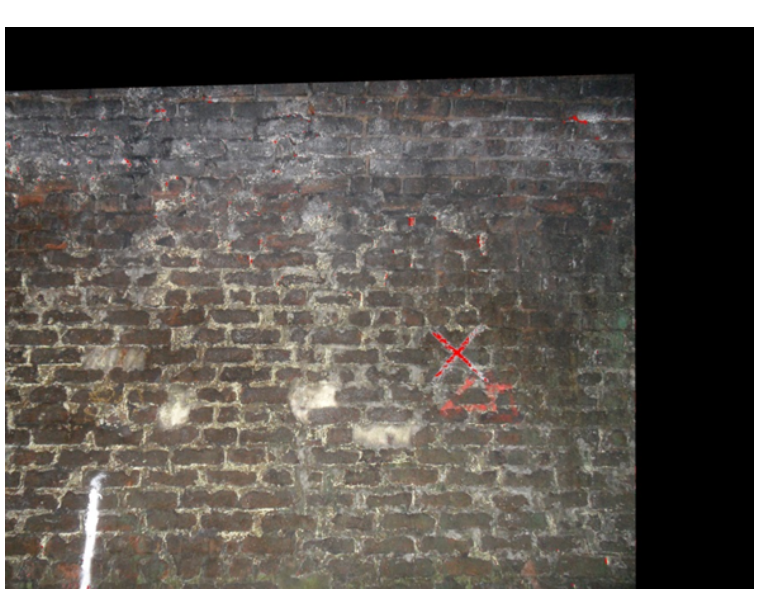

(e) Change Highlighted Image

Fig. 6. An example of the stages of the change detection algorithm

as changes. These false positive pixels are reduced as much as possible using a range of morphological operations. This results in a binary mask where black pixels indicate areas which are unchanged between the images and white pixels indicate change (Figure 6(d)).

The mask indicating areas of change is then overlaid on the query image to highlight changes to the examiner (Figure 6(e)). This reduces the time taken to inspect the image data as areas of interest are automatically detected and displayed. The ability to directly compare images of a section of tunnel from current and previous inspections is also of great value to examiners and allows for better analysis of the severity of a defect and the rate at which it is developing.

\section{3D Tunnel Reconstruction}

One benefit of the automated inspection methods which utilise laser scanning [1]-[3] is the ability to use the point cloud generated to create a 3D model of the structure which is being inspected. While a laser scanner gives a very accurate point cloud which shows the shape of the structure, image data must still be mapped to the point cloud to achieve a realistic rendering of the structure. Although the highly precise 


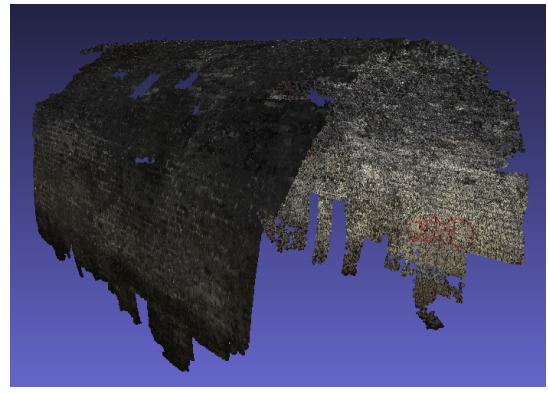

(a) Point Cloud

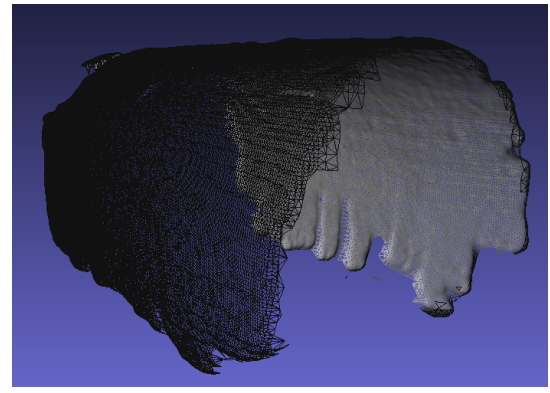

(b) Mesh Model

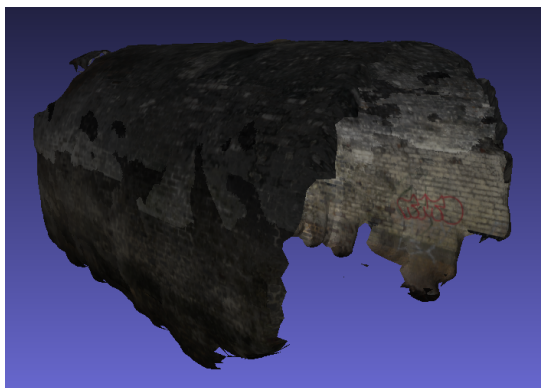

(c) Texture Mapped Model from Outside

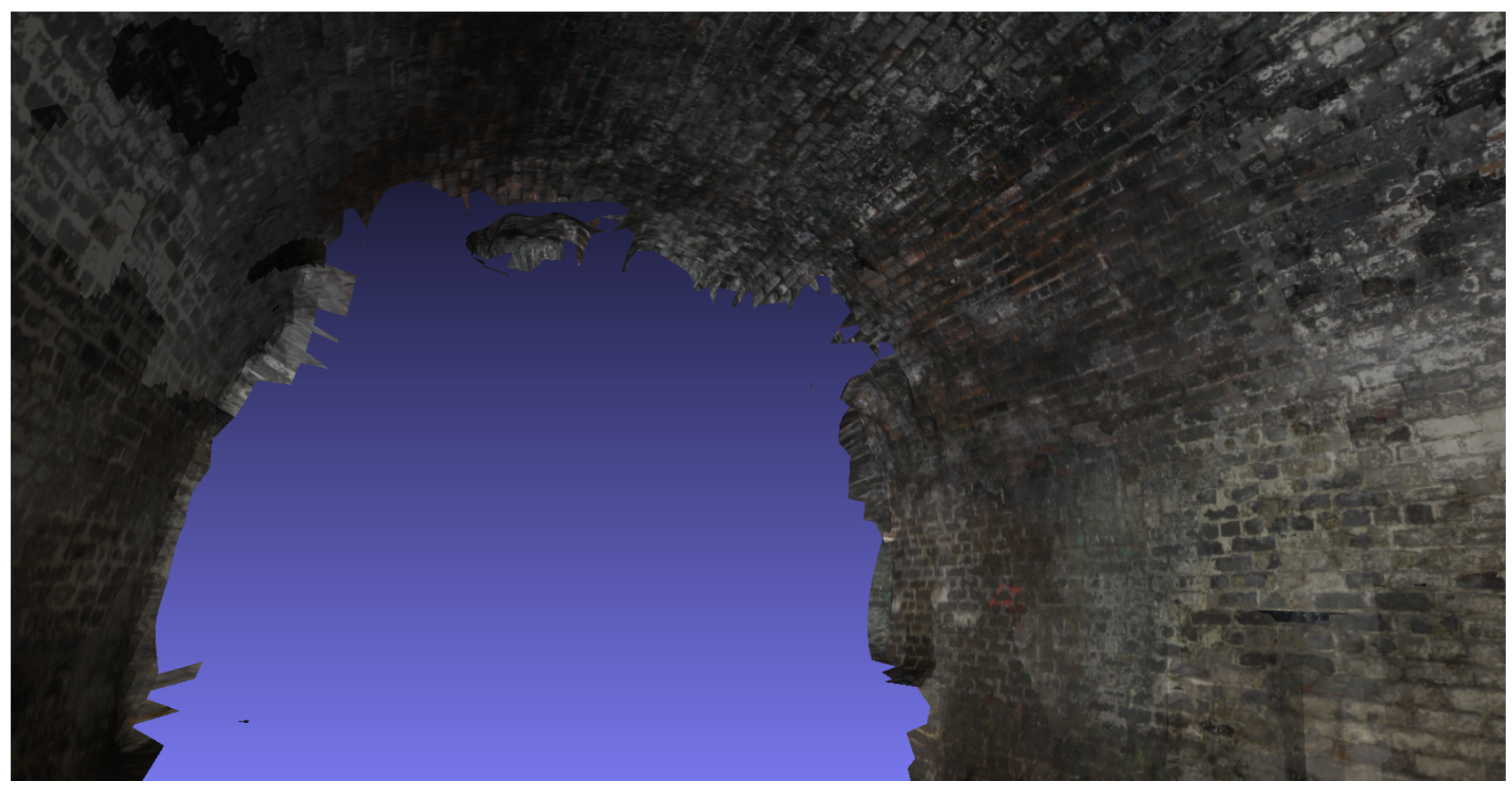

(d) Texture Mapped Model from Inside

Fig. 7. A point cloud, mesh model and final texture mapped 3D model generated using Structure from Motion

measurement associated with laser scanning is not necessarily required for tunnel inspection, it is still beneficial to generate a 3D model of the structure for use by examiners and to present to clients who may be interested in viewing the assets they own.

3D models of sufficient accuracy can be achieved from image data alone, without the requirement for expensive laser scanning equipment, using Structure from Motion (SfM) techniques. The work in [9] and [4] utilise SfM techniques but, as their operation is confined to cylindrical tunnels, the image data is assumed to map to a cylindrical mesh which simplifies the problem. As the system presented in this paper is designed to work on a much broader range of tunnel geometries, the SfM algorithm cannot be confined to a single geometric model.

To achieve this more generic 3D modelling capability we utilise an SfM algorithm which does not rely on a geometric prior [10]-[13]. This system requires only the input images from which a dense point cloud (shown in Figure 7(a)) is generated based on the change in position of the cameras between the capture of each image.
This point cloud is then processed further to create a mesh frame as shown in Figure 7(b). To complete the 3D reconstruction the images utilised to generate the point cloud must be texture mapped [14] onto the mesh frame. This results in a realistic reconstruction of the structure without the requirement of laser scanning as shown in Figure 7(c) which shows the full model and Figure 7(d) which shows a section of the model from the inside.

The resultant 3D model is once again useful to examiners in terms of contextualising the location of defects within the structure. It is also useful for supplying clients with reports detailing the structure condition which are more understandable.

\section{CONCLUSION}

The work presented in this paper is a complete system designed to aid structural examiners in the condition monitoring of railway tunnels. The system consists of both hardware and software elements and aims to reduce the time taken to carry 
out the visual inspection while increasing the accuracy and robustness.

The hardware system consists of an overlapping array of cameras and a uniform lighting solution which is mounted on a trolley. This trolley is moved through the tunnel collecting image data of the lining of the structure, decreasing the time required on site by removing the need for scaffolding to reach difficult locations.

The software package contains several elements. An image registration algorithm takes the individual images captured by the hardware system and creates a single stitched image of the tunnel lining. This allows examiners to view defects in the context of their location. A change detection algorithm is also implemented to direct the attention of examiners to important areas. The examiner can also view the defect at the time of the previous inspection to assess its rate of development. Finally, a 3D model of the structure is generated using SfM techniques. This again aids examiners in contextualising the location of defects and offers a unique reporting style to clients.

\section{ACKNOWLEDGEMENTS}

The work presented in this paper has been carried out in association with Geckotech Solutions Ltd who provide specialist access solutions to the railway and construction engineering industries. The authors would like to thank Geckotech Solutions Ltd for their support.

\section{REFERENCES}

[1] T. Ortner, G. Paar, G. Hesina, R.F. Tobler and B. Nauschnegg, "Towards true underground infrastructure surface documentation," 2010.

[2] "Spacetec," http://www.spacetec.de, 2012.

[3] R. Marani, M. Nitti, E. Stella, and T. DOrazio, "Monitoring of indoor environments by change detection in point clouds," in Environmental, Energy, and Structural Monitoring Systems (EESMS), 2016 IEEE Workshop on, pp. 1-6, IEEE, 2016.

[4] S. Stent, R. Gherardi, B. Stenger, K. Soga, and R. Cipolla, "An imagebased system for change detection on tunnel linings," in MVA, pp. 359$362,2013$.

[5] M. Brown and D. G. Lowe, "Automatic panoramic image stitching using invariant features," in International Journal of Computer Vision, vol. 74, no. 1, pp. 59-73, Springer, 2007.

[6] C. Tarantino, P. Blonda, and M. Adamo, "An application of the crosscorrelation analysis to detect changes in semi-natural grasslands to artificial structures using very high and high resolution satellite data," in Environmental, Energy, and Structural Monitoring Systems (EESMS), 2016 IEEE Workshop on, pp.1-6, IEEE, 2016.

[7] R. J. Radke, S. Andra, O. Al-Kofahi, and B. Roysam, "Image change detection algorithms: a systematic survey," in IEEE Transactions on Image Processing, vol. 14, no. 3, pp. 294-307, IEEE, 2005.

[8] B. Zitova and J. Flusser, "Image registration methods: a survey," in Image and Vision Computing, vol. 21, no. 11, pp. 977-1000, Elsevire, 2003.

[9] K. Chaiyasarn, T.-K. Kim, F. Viola, R. Cipolla, and K. Soga, "Image mosaicing via quadric surface estimation with priors for tunnel inspection," in Image Processing (ICIP), 2009 16th IEEE International Conference on, pp. 537-540, IEEE, 2009

[10] C. Wu, "Towards linear-time incremental structure from motion," in 3DTV-Conference, 2013 International Conference on, pp. 127-134, IEEE, 2013.

[11] C. Wu et al., "VisualSFM: A visual structure from motion system," http://ccwu.me/vsfm/, 2011.

[12] C. Wu, S. Agarwal, B. Curless, and S. M. Seitz, "Multicore bundle adjustment," in Computer Vision and Pattern Recognition (CVPR), 2011 IEEE Conference on, pp. 3057-3064, IEEE, 2011.

[13] C. Wu, "SiftGPU: A GPU implementation of scale invariant feature transform (SIFT)," http://cs.unc.edu/ ccwu/siftgpu, 2007.

[14] N. Ho, "TextureMesh," http://nghiaho.com/?page_id=1629, 2013. 